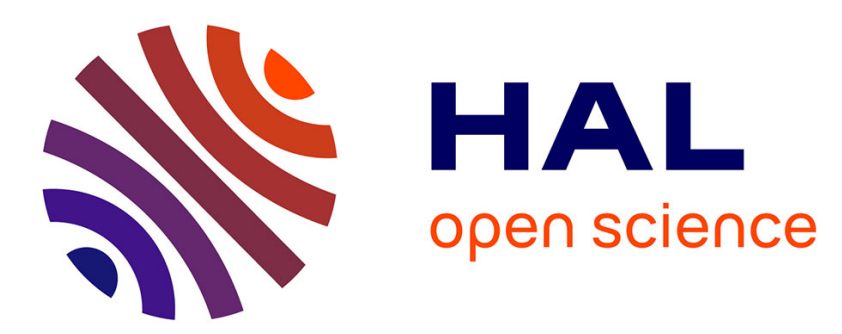

\title{
Effet de la taille et de la forme des grains sur quelques caractéristiques du développement du maïs au stade jeune
}

Nabil Abd El Rahman, Robert Bourdu

\section{- To cite this version:}

Nabil Abd El Rahman, Robert Bourdu. Effet de la taille et de la forme des grains sur quelques caractéristiques du développement du maïs au stade jeune. Agronomie, 1986, 6 (2), pp.181-186. hal00884863

\section{HAL Id: hal-00884863 \\ https://hal.science/hal-00884863}

Submitted on 1 Jan 1986

HAL is a multi-disciplinary open access archive for the deposit and dissemination of scientific research documents, whether they are published or not. The documents may come from teaching and research institutions in France or abroad, or from public or private research centers.
L'archive ouverte pluridisciplinaire HAL, est destinée au dépôt et à la diffusion de documents scientifiques de niveau recherche, publiés ou non, émanant des établissements d'enseignement et de recherche français ou étrangers, des laboratoires publics ou privés. 


\title{
Effet de la taille et de la forme des grains sur quelques caractéristiques du développement du maïs au stade jeune
}

\author{
Nabil ABD EL RAHMAN \& Robert BOURDU \\ Laboratoire «Structure et Métabolisme des Plantes » associé au C.N.R.S. (U.A. 1128), Université de Paris- \\ Sud (XI), F 91405 Orsay Cedex
}

\begin{abstract}
Dans un travail antérieur (BOURDU \& GRÉGORY, 1983), nous avions montré que plusieurs paramètres du début de la croissance du maïs pouvaient servir à une comparaison intervariétale. On a utilisé le même type d'analyse pour comparer l'effet du calibre et de la forme des grains d'une même variété de maïs (LG 11) sur les caractéristiques du début de la croissance. On constate que les plantules issues des grains les plus gros manifestent un taux de croissance plus élevé ; il en est de même pour celles provenant des grains ronds. Cependant, si la plupart des caractères sont affectés, il en est un qui reste constant : c'est celui qui mesure l'efficacité d'utilisation des réserves séminales pour l'édification d'un équipement photosynthétique fonctionnel. Ce caractère peut donc être considéré comme constante variétale.
\end{abstract}

Mots clés additionnels : Zea mays, germination, vigueur germinative.

\section{The effect of seed size and shape on early development in maize.}

In a previous paper in this series, we showed (BOURDU \& GREGORY, 1983) that several parameters of early maize growth could be useful for intervarietal comparisons. We have now used the same type of analysis to study the effect of grain size and shape in one variety of maize (LG 11). We found that large grains are associated with higher growth rate and that round forms produce better seedlings than flat ones. Most of these characters are affected by grain size and shape, but one of them is constant in the four grain categories, i.e. the efficiency of utilization of maternal reserves for building the photosynthetic apparatus. Thus this criterion can be considered as a genotypic characteristic.

Additional key words : Zea mays, germination, seedling vigour.

\section{INTRODUCTION}

Il existe dans la littérature un assez large consensus pour admettre que la taille d'une semence influe favorablement sur les qualités de la jeune plante issue de la germination, voire sur la croissance et la production finale.

Les études publiées, et certaines sont très anciennes (JeTHRo TUlL, 1733 ; KIESSELBACH \& Helm, 1917), portent principalement - mais non exclusivement sur des légumineuses cultivées telles que le trèfle (BLACK, 1957 ; Williams et al., 1968) ou le soja (FEHR \& PROBST, 1971 ; FONTES \& OHLROGGE, 1972 ; BURRIS et al., 1973) ou sur des céréales telles que le blé (KITTOCK \& LAW, 1968; AUSTENSON \& WALTON, 1970 ; RIES \& EVERSON, 1973 ; PURI \& QUALSET, 1978 ; HAMPTON, 1981), le sorgho (ABDUL-
LAHI \& VANDERLIP, 1972), l'orge (DEMIRLICAKMAK et al., 1963; KAUFMANN \& GUITARD, 1967) ou le maïs (HAMMES, 1969 ; HUNTER \& KANNENBERG, 1972 ; BURRIS, 1977 ; GEORGIEV et al., 1979). Les corrélations sont établies entre le poids ou le volume des semences et les manifestations précoces du développement de la jeune plante telles que le pouvoir germinatif et la vitesse de germination ou la vigueur de la plantule en début de végétation. Quand la corrélation est significative, on peut dire que la taille de la semence fait partie du descriptif de cette notion souvent utilisée bien qu'ambiguë de "qualité de la semence ").

Mais il y a plus, dans la mesure où certains travaux (ClARK \& PECK, 1968 : FEHR \& PROBST, 1971 ; HUNTER \& KANNENBERG, 1972; AHMED \& ZUBERI, 1973 ; PURI \& QUALSET, 1978 ; GEORGIEV et al., 
1979) montrent que la taille de l'investissement initial (la semence) se répercute en fin de chaîne, c'est-à-dire à la récolte, on doit considérer que l'effet «qualité taille » peut dépasser dans ses conséquences les seules manifestations physiologiques juvéniles. Les explications doivent alors prendre en compte l'effet durable d'une manifestation précoce.

Nous avons décrit dans un travail antérieur (BOURDU \& GRÉGORY, 1983) une série d'événements physiologiques qui se situent au cours des phases de germination, de levée et de croissance au stade jeune du maïs cultivé en conditions contrôlées. Ces événements quantifiés permettent d'appréhender des caractéristiques métaboliques générales. Des variations intervariétales ont été montrées. Nous avons émis l'hypothèse que certains de ces caractères marquaient de leur influence directe ou indirecte les propriétés ultérieures de la plante (précocité, résistance aux stress, productivité...).

Les résultats qui sont exposés ici ont été obtenus en utilisant une même approche méthodologique mais, cette fois-ci, pour procéder à une comparaison intravariétale ( $L G$ 11), la variable étant le poids et la forme des grains. On montre que le poids des semences impose une cinétique de croissance initiale propre à chaque calibre avec un taux de croissance diminué pour les plus faibles tailles mais que d'autres caractéristiques telles que la vitesse de mise en place d'un appareil photosynthétique efficace ne semblent pas affectées. Cependant, dans les limites de l'expérience, les qualités « taille » et « forme initiale » des semences pèsent d'un poids de plus en plus lourd en fonction du temps traduisant un effet cumulatif évident.

\section{MATÉRIEL ET MÉTHODES}

Le travail a été réalisé sur le génotype de maïs " $\mathrm{LG} 11$ » dont les semences ont été réparties en 4 lots caractérisés par leur poids et leur forme :

- Les semences petites et plates $(=P P)$, poids moyen de $215 \mathrm{mg}$.

- Les semences petites et rondes ( $=P R)$, poids moyen de $243 \mathrm{mg}$.

- Les semences grosses et plates ( $=$ GP), poids moyen de $313 \mathrm{mg}$.

- Les semences grosses et rondes ( $=\mathrm{GR})$, poids moyen de $352 \mathrm{mg}$.

Ces semences ont été fournies par la Station d'Amélioration des Plantes, I.N.R.A., de Mons-enChaussée.

L'expérience reprend les modalités décrites dans le travail de BOURDU \& GRÉGORY (1983) ; on rappellera ici seulement les principes.

Les semences individuellement pesées ont été mises pour germination et développement dans des pots individuels remplis de vermiculite imbibée de solution nutritive (solution BB $^{\prime}$ de Coïc). Les cultures ont été placées en enceinte climatisée $\left(25^{\circ} \mathrm{C}\right.$ le jour et $22^{\circ} \mathrm{C}$ pendant la période obscure de $8 \mathrm{~h} / 24 \mathrm{~h}$ ). L'éclairement maintenu au niveau des apex pendant la photopériode $(16 \mathrm{~h} / 24 \mathrm{~h})$ était de $220 \mu \mathrm{M} \cdot \mathrm{m}^{-2} \cdot \mathrm{s}^{-1}$. A partir du $3^{\mathrm{e}}$ jour après le début de l'imbibition (temps 0 ) et chaque jour, 6 plantes ont été prélevées toujours à la mềme heure de la journee et suikant un plan de rando. misation. Les plantules, d'une part, et le reste de la semence, d'autre part, ont été séparés puis pesés avant et après déshydratation complète $\left(80^{\circ} \mathrm{C}\right.$ pendant $48 \mathrm{~h}$ ). Les expériences de culture ont été répétées 3 fois sauf pour le lot GR qui n'a fait l'objet que de 2 expériences.

\section{RÉSULTATS ET DISCUSSION}

Quand on considère les cinétiques de croissance des plantules issues des 4 lots de semences (fig. 1A), on constate qu'il existe une différence évidente et significative entre les plantules provenant des grosses semences et celles issues des petites semences. Les différences entre les formes rondes et les formes plates dans les 2 catégories de tailles ne sont significativement

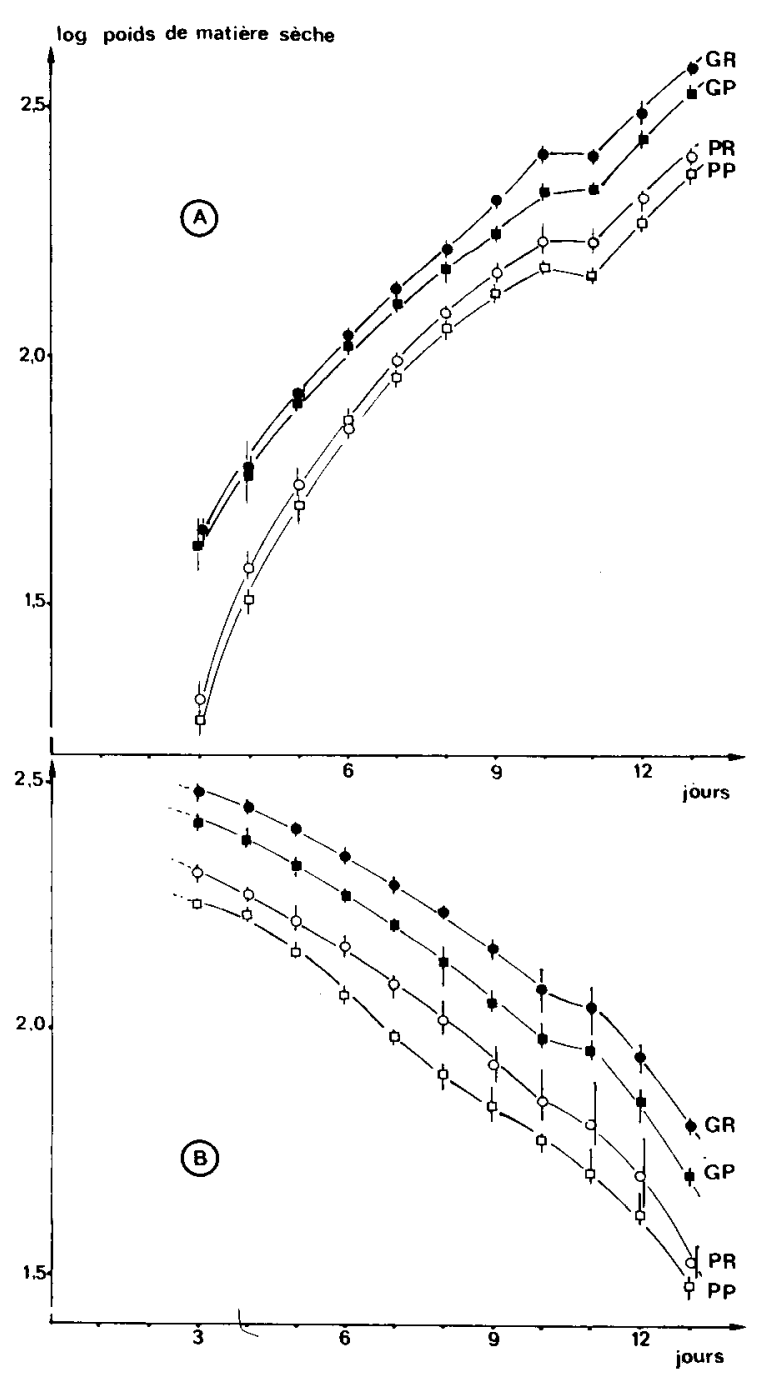

Figure 1

Courbes de croissances en coordonnées semi-log des jeunes plantes de mais issues de différents lots de semences (variété LG I1).

Growth curves (semi-logarithmic scale) for maize seedlings from various seed categories (variety LG 11).

A. Croissance de la plantule; seedling growth.

$B$. Disparition des réserves; reserve decrease.

Chaque point représente la moyenne de 3 expériences, sauf pour GR où il s'agit de la moyenne effectuée sur 2 expériences. Les barres relient les valeurs extrêmes enregistrées.

(Every point represents the average of three experiments except for $G R$ which is the average of two experiments. Bars show the extreme vuhes in eoch case.) 
marquées qu'à partir du $6^{\mathrm{e}}$ ou $7^{\mathrm{e}}$ jour. Cependant, on peut noter que tout au long des courbes les points représentatifs des lots ronds sont systématiquement, bien que légèrement, au-dessus des valeurs obtenues à partir des lots plats. Cette différence peut ne pas être due simplement à la forme mais prolonger la différence due au poids puisqu'au sein des lots " grosses semences » et « petites semences », systématiquement, les formes rondes sont plus lourdes que les formes plates. La linéarité des ajustements de la figure 3 iraient bien dans ce sens.

On retrouve, parfaitement illustrée, la crise qui, dans nos conditions expérimentales, se situe ici au $11^{\mathrm{e}}$ jour. A cette date, la croissance cesse transitoirement. Cet événement a été observé par BouRdu \& GRÉGORY (1983) et analysé sur le plan physiologique par DELÉENS et al. (1984). Il s'agit du moment où, au cours du développement de la jeune plante, se met en place le système de translocation qui assure la nutrition carbonée des racines à partir du carbone assimilé dans les jeunes feuilles. Cette crise dans la croissance semble affecter de façon égale les 4 lots de plantes. Une vérification quantitative est possible ; on peut, en effet, avoir une approche de l'importance de cette cassure dans la cinétique de croissance en calculant la différence qui existe entre la valeur effectivement enregistrée le jour de cette crise (le $11^{\mathrm{e}}$ jour, ici) et une valeur théorique obtenue en faisant la moyenne entre le jour précédent et le jour suivant, c'est-à-dire comme si cette crise n'existait pas. On fait alors $l^{\prime h y p o t h e ̀ s e ~ d ' u n e ~ c r o i s s a n c e ~ l i n e ́ a i r e ~ e n t r e ~ l e ~} 10^{\mathrm{e}}$ et le $12^{\mathrm{e}}$ jour, ce qui est admissible compte tenu des valeurs enregistrées juste avant et immédiatement après. Le " manque à gagner " au cours de cette $11^{\mathrm{e}}$ journée est toujours plus élevé pour les plantes issues de grosses semences que pour les plantes issues de petites semen-

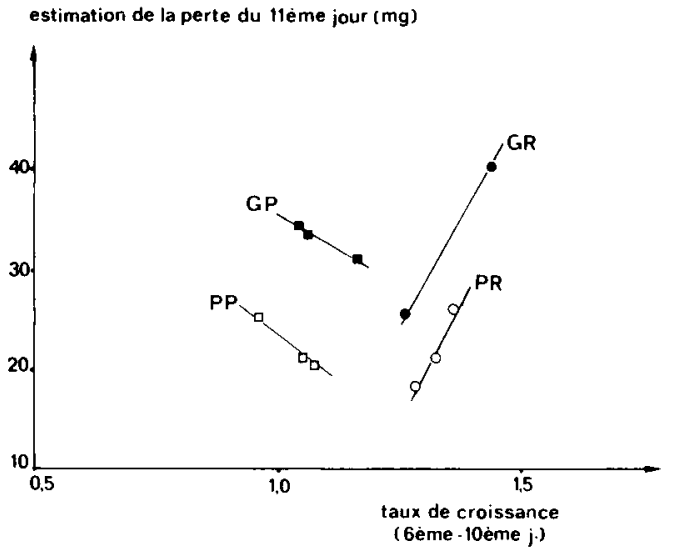

Figure 2

Relations entre la taille et la forme des semences et l'importance estimée de l'arrêt de la croissance de la plantule observé au $11^{e}$ jour.

Relations between the size and the form of the seeds and the estimated importance of seedling growth arrest observed at 11th day.

ces. Cependant, la figure 2 permet d'apporter de nouvelles précisions. Dans la mesure où le nombre limité d'expériences permet une conclusion, on pourrait penser qu'une corrélation existerait entre le taux de croissance enregistré juste avant l'accident et l'importance de cet accident. Cette corrélation serait négative dans le cas des semences plates et positive dans le cas des semences rondes. Cette remarque, si elle était confirmée par de plus nombreuses observations, trouverait sans doute son explication dans la structure différente des embryons situés dans les 2 types de grains.

On peut évaluer l'incidence de la variable poids de la semence sur la taille de la plantule grâce au graphique de la figure 3 . Sur cette figure, le poids de la

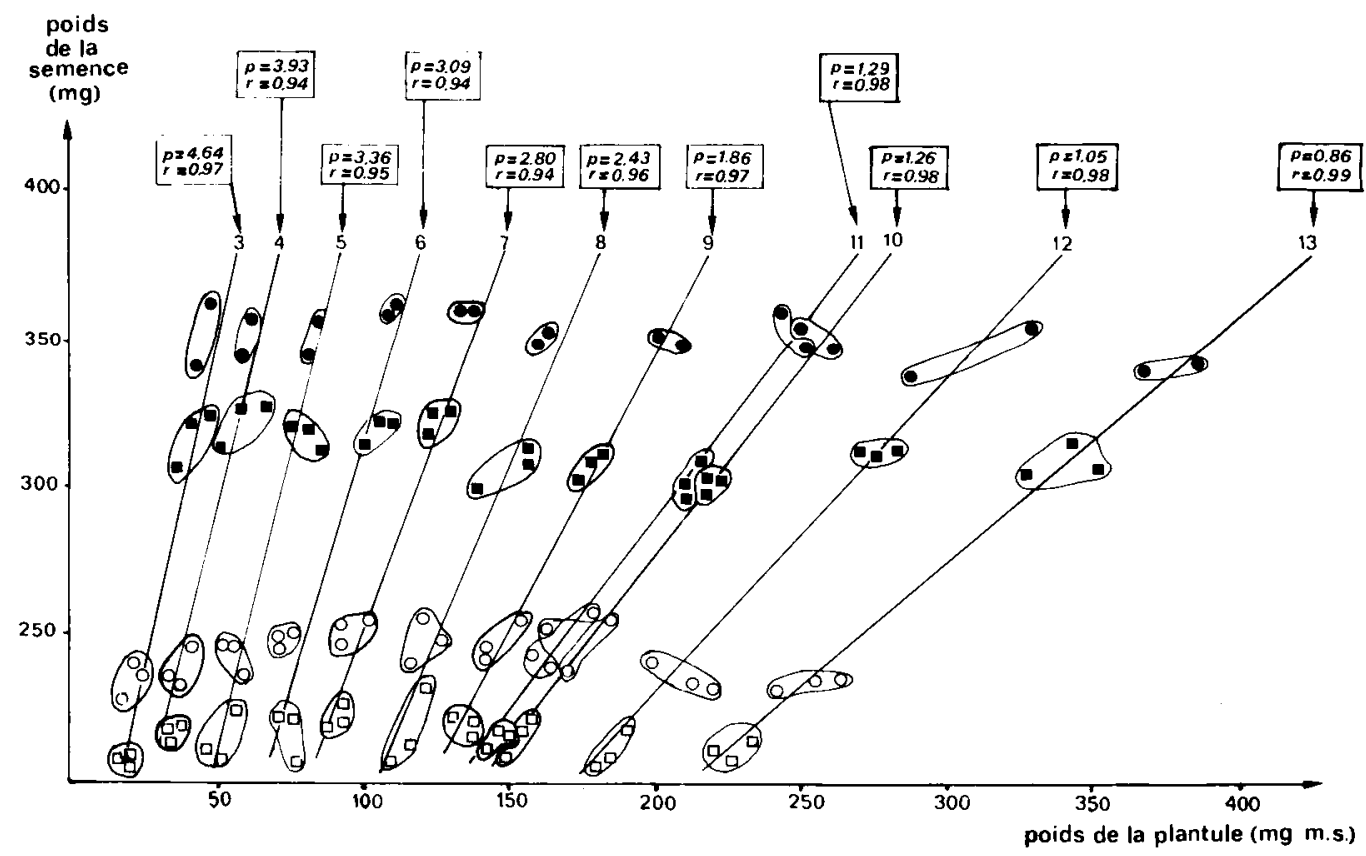

Figure 3

Corrélations entre le poids de la plantule âgée de 3 à 13 jours et le poids de la semence.

Correlations between seed weight and the weight of three to thirteen day-old seedlings.

$\begin{array}{ll}-G R & O P R \\ G P & \square P P\end{array}$ $p=$ pente de la droite de corrélation; slope of the correlation line. $r=$ coefficient d'ajustement linéaire; coefficient of linear adjustement.

Les nombres placés au-dessus des droites indiquent l'âge de la plantule.

Number above each line indicates the age of the seedling. 
plantule à un âge donné, $3,4,5$ jours, etc... (abscisse) est mis en corrélation avec le poids initial de la semence (ordonnée) ; chacun des 4 lots est limité par une courbe fermée et une droite d'ajustement linéaire est tracée pour chaque jour prenant en compte la totalité des 11 cultures ( 3 répétitions par lot sauf 2 pour le lot GR).

On constate, mais cela était déjà perceptible sur la figure $1 \mathrm{~A}$, qu'en prenant de l'âge, la plantule profite de plus en plus du gain initial de taille. Au cours des premiers jours ( $3^{\mathrm{e}}$ et $4^{\mathrm{e}}$ jours), la pente de la droite de corrélation traduit simplement le rapport pondéral entre l'albumen et les téguments, d'une part, et l'embryon, d'autre part, mais, par la suite, on assiste à une sommation des effets et l'avantage s'amplifie au profit des jeunes plantes issues des graines les plus grosses. On peut remarquer qu'au $11^{\mathrm{e}}$ jour, jour de la crise, la droite de corrélation a une origine dont le décalage traduit simplement la légère régression de la croissance, mais sa pente est rigoureusement identique à celle du $10^{\mathrm{e}}$ jour, ce qui signifie que la crise de croissance marque un retard dans l'évolution du développement.

La figure 1B montre la cinétique de décroissance des réserves de la semence. On y retrouve les différences significatives entre les lots ainsi que l'accident du $11^{\mathrm{e}}$ jour où le ralentissement de l'épuisement des réserves est plus marqué pour les grosses semences que pour les petites. Dans les limites de l'expérience, l'utilisation des réserves est plus profonde pour les petites semences que pour les grosses (fig. 4).

Nous avions montré qu'une autre représentation de la croissance pouvait apporter de nouvelles informations (BOURDU \& GRÉGORY, 1983). Cette représentation illustrée par la figure 5 donne, pour chaque jour, le rapport entre le poids total de la germination (plantule + réserves) mesuré à ce jour et le poids initial de la semence. On avait insisté sur la durée de la phase durant laquelle ce rapport reste inférieur à 1 et la concavité de cette phase. On constate sur la figure 5 qu'il apparaît une différence entre les lots, principalement entre les grosses et les petites semences. Alors que les grosses semences produisent des plantes qui acquièrent

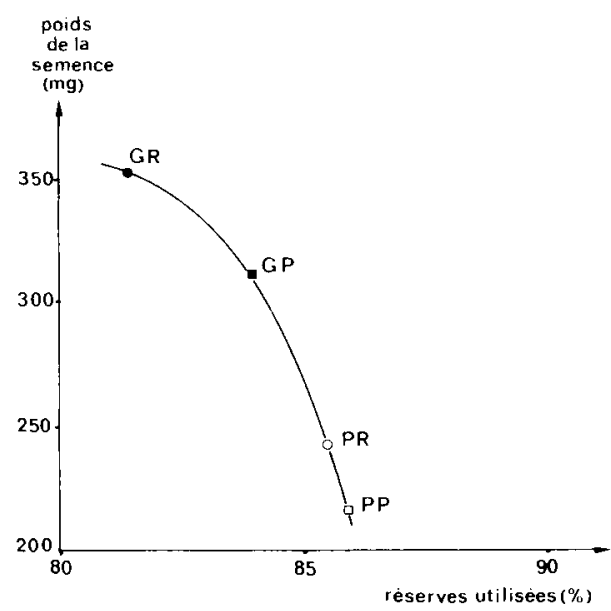

Figure 4

Relation entre la quantité de réserves utilisées en 13 jours et le poids de la semence.

Relation between the quantity of reserves consumed in thirteen days and seed weight.

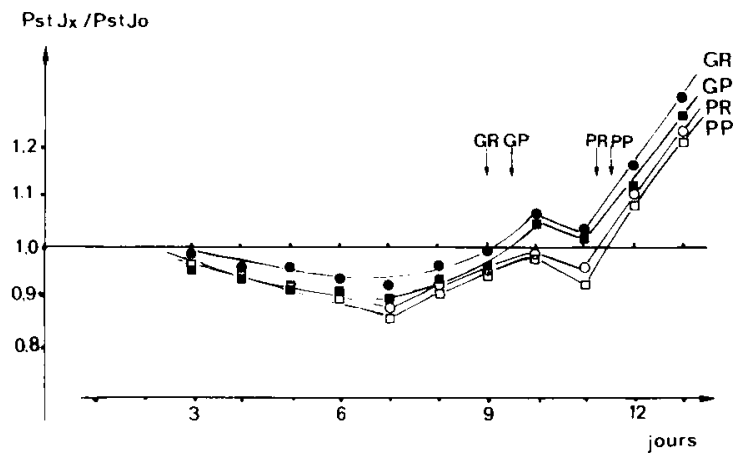

Figure 5

Evolution du rapport : poids de matière sèche totale $\left(P_{s} t J_{x}\right) /$ poids de matière sèche initiale de la semence $\left(P_{s} t J_{o}\right)$. Les flèches indiquent pour chaque lot le moment où le rapport est égal à $l$.

Variation in the ratio : total seedling dry weight at day $J_{x}\left(P_{s} t J_{y}\right) /-$ initial seed dry weight $\left(P_{s} t J_{o}\right)$. The arrows show for each category the moment that ratio equals 1 .

un poids égal ou supérieur au poids initial (rapport $\geqslant 1)$ le $9^{e}$ jour, les autres ne dépassent cette limite qu'entre le $11^{\mathrm{e}}$ et $12^{\mathrm{e}}$ jour. Ce retard est dû au fait que la crise de croissance, qui est bien visible sur ce genre de représentation, maintient plus longtemps les jeunes plantes issues de petites semences dans la partie d'ordonnée $<1$. Cette aggravation du retard initial peut porter un préjudice important et durable au développement ultérieur de la plante.

On observe que dans la partie concave des courbes, dans la zone $<1$, la diminution du rapport qui correspond à la période pendant laquelle les pertes respiratoires ne sont pas compensées par une activité photosynthétique efficace cesse très exactement au même moment pour tous les lots, le $7^{\mathrm{e}}$ jour dans nos conditions expérimentales. Cela signifie que le temps nécessaire pour créer un appareil photosynthétique apte à compenser les activités de perte en carbone est indépendant de la taille et de la forme de la semence.

Quand on porte sur un graphique les poids de matière sèche de la plantule au cours du temps en fonction de la quantité (relative ou absolue) de réserves utilisées, on constate que le graphe possède 2 parties (fig. 6) : une $1^{\text {re }}$ partie où il y a correspondance linéaire entre les réserves utilisées et la prise de poids de la jeune plante et une $2^{\mathrm{e}}$ partie où la croissance pondérale échappe à cette liaison et où il n'y a plus de rapport constant entre les pertes des réserves et les gains de la plantule. On peut penser qu'alors les activités autotrophiques ont pris le relais et l'avantage sur la nutrition hétérotrophique aux dépens des réserves carbonées séminales. La figure 6 illustre ces relations pour les 4 lots de l'expérience. On constate des différences et une constante. Les différences portent sur la $1^{\text {re }}$ partie des graphes. Les pentes des droites sont très nettement supérieures pour les plantules provenant des grosses semences à celles provenant des petites. La pente traduit l'efficacité d'utilisation des réserves, une forte pente indiquant un rendement élevé de transfert et d'utilisation pour l'édification des nouveaux tissus. Les grosses semences sont donc favorisées sous cet aspect.

Mais la figure 6 montre une constante pour les 4 lots. C'est toujours pour la même proportion de réserves utilisées que la liaison entre les coordonnées 


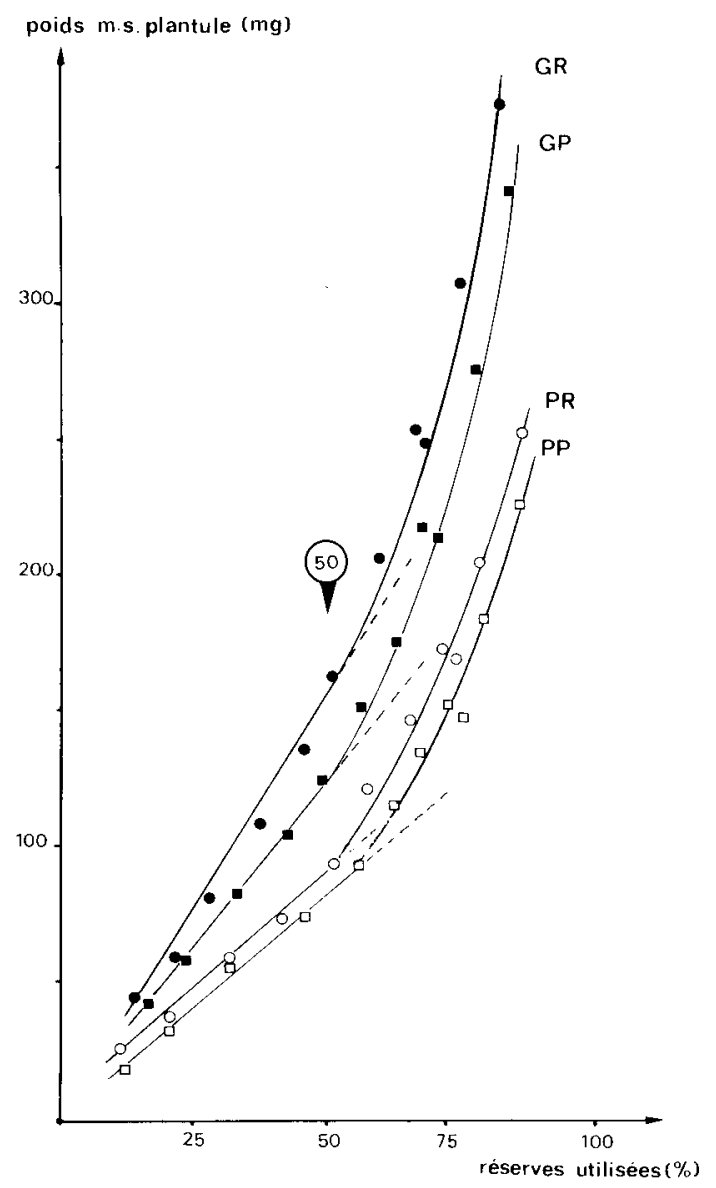

Figure 6

Corrélations entre les quantités de réserves utilisées et le poids des plantules issues des différents lots de semence. La flèche « 50 » souligne le pourcentage de réserves utilisées à partir duquel la croissance de la plantule échappe au contrôle absolu et constant de la quantité des réserves métabolisées.

Correlations between the amount of reserve consumed and seedling weight for various seed categories. The arrow « 50 " points out the percentage of reserve consumed at which time the growth is no longer under the dependence of metabolic reserves.

échappe à la linéarité. Cette valeur est égale à 50 p. 100. C'est cette même valeur que l'on avait trouvée pour «LG 11 » dans le travail antérieur (BOURDU \& GRÉGORY, 1983). On avait montré que cette valeur pouvait varier d'un génotype à l'autre. Le fait que, quelles que soient les qualités de la semence, c'est toujours à 50 p. 100 des réserves consommées que la croissance des jeunes plantes de "LG 11 » échappe au contrôle séminal confirme qu'il s'agit d'un caractère génétique.
Les différences de poids et de taille entre les semences de maïs tiennent, pour un génotype donné et pour une récolte donnée, à la position du grain sur l'épi dans la mesure, bien entendu, où l'échantillonnage en épis n'a pas introduit une variabilité interplante quant au taux de fécondation et donc de richesse en grains des épis. L'homogénéité interplante nous a été assurée par le fournisseur du matériel biologique. S'il en est ainsi, la position des grains impose 2 types de contraintes ou de contrôles sur leur développement : une limite de l'espace disponible à l'expansion (grains plats et comprimés ou grains ronds) ou une limite de l'approvisionnement en métabolites lors du remplissage (grains favorisés à la base par rapport au sommet). De ces 2 types de contraintes, découlent 2 sortes de réponses possibles : dans le $1^{\text {er }}$ cas, l'expansion de l'embryon peut être freinée par rapport au remplissage des tissus de réserve, dans l'autre cas la nature, la qualité et la quantité des réserves peuvent être plus affectées que la mise en place des structures embryonnaires. Les petites graines (plates ou rondes) sont situées au sommet de l'épi et donc défavorisées quant à l'accumulation des réserves; elles donnent toujours des plantules moins lourdes et cet handicap au départ va en s'aggravant. Les graines plates (petites ou grosses) ont un développement déséquilibré entre les 2 compartiments (réserves et plantules) et cela se traduit par un désavantage dans le développement initial de la plante par rapport aux graines rondes. C'est ce qui ressort des résultats présentés ici. Que par la suite le décalage entre les plantes issues des différents types de semences s'estompe est un autre problème. Mais les différences que nous avons montrées portent sur une période de la vie du maïs où toute pénalité peut être majorée par l'effet d'un microclimat défavorable. Les effets risquent alors d'être cumulatifs.

Reçu le 12 juillet 1985. Accepté le 10 octobre 1985.

\section{REMERCIEMENTS}

Nous remercions la Station d'Amélioration des Plantes de I'I.N.R.A. à Estrées-Mons (Directeur : Monsieur DERIEuX) par l'intermédiaire de laquelle les semences nous ont été fournies, la Société Limagrain en étant productrice. Nous associons cette société à nos remerciements. Nous remercions également Madame D. GILLION pour son aide technique. 


\section{REFERENCES BIBLIOGRAPHIQUES}

Abdullahi A., Vanderlip R. L., 1972. Relationships of vigor tests and seed source and size to Sorghum seedling establishment. Agron. J., 64, 143-144.

Ahmed S. U., Zuberi M. I., 1973. Effects of seed size on yield and some of its components in rape-seed (Brassica campestris). Crop Sci., 13, 119-120.

Austenson H. M., Walton P. D., 1970. Relationships between initial seed weight and mature plant characters in spring wheat. Can. J. Plant Sci., 50, 53-58.

Black J. N., 1957. The early vegetative growth of three strains of subterranean clover (Trifolium subterraneum) in relation to seed size. Aust. J. Agric. Res., 8, 1-14.

Bourdu R., Grégory N., 1983. Etude comparée du début de la croissance chez divers génotypes de maïs. Agronomie, 3 (8), 761-770.

Burris J. S., 1977. Effect of location of production and maternal parentage on seedling vigour in hybrid maize. Seed Sci. Technol., 5 (4), 703-708.

Burris J. S., Edje T. J., Wahab A. H., 1973. Effects of seed size on seedling performance in soybeans. II. Seedling growth and photosynthesis and field performance. Crop Sci., 13, 207-210.

Clark B. E., Peck N. H., 1968. Relationship between the size and performance of snap bean seeds. N.-Y. State Agric. Exp. Stn. Bull. 819.

Deléens E., Grégory N., Bourdu R., 1984. Transition between seed reserve use and photosynthetic supply during development of maize seedlings. Plant Sci. Lett., 37, 35-39.

Demirlicakmak A., Kaufmann M. I., Johnson L. P. V., 1963. The influence of seed size and seedling rate on yield and yield components of barley. Can. J. Plant Sci., 43, 330-337.

Fehr W. R., Probst A. H., 1971. Effect of seed source on soybean strain performance for two successive generations. Crop Sci., 11, 865-867.
Fontes L. A. N., Ohlrogge A. J., 1972. Influence of seed size and population on yield and other characteristics of soybean. Agron. J., 64, 833-836.

Georgiev T., Mukhtanov I., Angelova L., 1979. Correlation between grain yield, stalk strength, protein and lysine content in corn. Genet. Sel., 12 (1), 11-20.

Hammes P. S., 1969. Seed characteristics and seedling growth of graded maize seed. Agric. Sci. S. Afr. (I) Agroplantae (I), 1, 33-38.

Hampton J. G., 1981. The extent and significance of seed size variation in New Zealand wheats (Triticum aestivum). N.Z.J. Exp. Agric., 9 (2), 179-184.

Hunter R., Kannenberg, 1972. Effects of seed size on emergence, grain yield and plant height in corn. Can. J. Plant Sci., 52, 252-256. Jethro Tull., 1733. Horse-hoeing husbandry. London. 257 p.

Kaufmann M. L., Guitard A. A., 1967. The effect of seed size on early plant development in barley. Can. J. Plant Sci., 47, 73-78.

Kiesselbach T. A., Helm C. A., 1917. Relation of size of seed and sprout value to the yield of small grain crops. Nebr. Agric. Exp. Stn. Res. Bull. II.

Kittock D. L., Law A. G., 1968. Relationship of seedling vigor to respiration and tetrazolium chloride reduction by germinating wheat seeds. Agron. J., 60, 286.

Puri Y. P., Qualset C. O., 1978. Effect of seed size and seedling rate on yield and other characteristics of durum wheat. Phyton Rev. Int. Bot. Exp. 36 (1), 41-52.

Ries S. K., Everson E. H., 1973. Protein content and seed size relationships with seedling vigor of wheat cultivars. Agron. J., 65 884-886.

Williams W. A., Black J. N., Donald C. M., 1968. Effect of seed weight on the vegetative growth of competing annual Trifoliums. Crop Sci., 8, 660-663. 